\title{
Experimental Evidence of Helical Flow in Porous Media
}

\author{
Ye, Yu; Chiogna, Gabriele; Cirpka, Olaf A.; Grathwohl, Peter; Rolle, Massimo
}

Published in:

Physical Review Letters

Link to article, DOI:

10.1103/physrevlett.115.194502

Publication date:

2015

Document Version

Publisher's PDF, also known as Version of record

Link back to DTU Orbit

Citation (APA):

Ye, Y., Chiogna, G., Cirpka, O. A., Grathwohl, P., \& Rolle, M. (2015). Experimental Evidence of Helical Flow in Porous Media. Physical Review Letters, 115(19), [e137540]. https://doi.org/10.1103/physrevlett.115.194502

\section{General rights}

Copyright and moral rights for the publications made accessible in the public portal are retained by the authors and/or other copyright owners and it is a condition of accessing publications that users recognise and abide by the legal requirements associated with these rights.

- Users may download and print one copy of any publication from the public portal for the purpose of private study or research.

- You may not further distribute the material or use it for any profit-making activity or commercial gain

- You may freely distribute the URL identifying the publication in the public portal 


\title{
Experimental Evidence of Helical Flow in Porous Media
}

\author{
Yu Ye, ${ }^{1}$ Gabriele Chiogna, ${ }^{2,1}$ Olaf A. Cirpka, ${ }^{1}$ Peter Grathwohl, ${ }^{1}$ and Massimo Rolle ${ }^{3,1, *}$ \\ ${ }^{1}$ Center for Applied Geoscience, University of Tübingen, Hölderlinstraße 12, D-72074 Tübingen, Germany \\ ${ }^{2}$ Faculty of Civil, Geo and Environmental Engineering, Technical University of Munich, Arcistraße 21, D-80333 Munich, Germany \\ ${ }^{3}$ Department of Environmental Engineering, Technical University of Denmark, Miljøvej Building 115, DK-2800 Lyngby, Denmark
}

(Received 24 March 2015; published 4 November 2015)

\begin{abstract}
Helical flow leads to deformation of solute plumes and enhances transverse mixing in porous media. We present experiments in which macroscopic helical flow is created by arranging different materials to obtain an anisotropic macroscopic permeability tensor with spatially variable orientation. The resulting helical flow entails twisting streamlines which cause a significant increase in lateral mass exchange and thus a large enhancement of plume dilution (up to 235\%) compared to transport in homogenous media. The setup may be used to effectively mix solutes in parallel streams similarly to static mixers, but in porous media.
\end{abstract}

PACS numbers: 47.56.+r, 45.70.Mg, 47.15.G-, 92.40.K-

Mixing processes are widely studied in fluid mechanics due to their relevance for many industrial and environmental processes [1-4]. While in turbulent flows efficient mixing can be easily achieved [5], slow diffusive processes control mixing in laminar and creeping flows encountered in engineering systems such as chemical separators, chromatography columns, micromixers and filters, as well as in natural geologic formations like aquifers and oil reservoirs [6-8]. Under these conditions, the topology of the flow field and its complex structure are fundamental to enhance and control mixing processes $[1,6,9,10]$. Flows in porous media have been mainly analyzed focusing on the shearing effects of heterogeneity on scalar mixing [11-14]. Most studies have considered heterogeneous isotropic porous media, in which the flow has a zero helicity density; i.e., the flow vector is always perpendicular to the vorticity vector [15]. However, a few theoretical and numerical studies [16-18] have shown that helical flows with nonzero helicity density can occur in three-dimensional anisotropic porous media. Yet, an experimental proof of the real occurrence of such flows in porous media is still missing.

In this Letter, we present laboratory flow-through experiments in a three-dimensional heterogeneous, anisotropic packed bed that prove the existence of helical flows in porous media, and we quantify the impact of such flows on steady-state transport and dilution of solute plumes.

Figure 1 shows a photograph of the experimental setup. The three-dimensional flow-through chamber has an inner dimension of $30 \mathrm{~cm} \times 7.7 \mathrm{~cm} \times 10 \mathrm{~cm}$. It has 25 ports $(5 \times 5$ array $)$ at the inlet and 49 ports $(7 \times 7$ array $)$ at the outlet. The ports at the inlet are equally spaced with a distance of $1.54 \mathrm{~cm}$, while at the outlet the distance between the ports is reduced to $1.1 \mathrm{~cm}$ in order to increase the spatial resolution of solute concentration and flow rate measurements. Rubber septa are plugged at all ports and stainless-steel syringe needles are inserted into the septa for liquid injection and extraction. High-precision multichannel peristaltic pumps (Ismatec, Glattbrugg, Switzerland) are used to establish steady-state flow in the flow-through setup. The pumping rates are individually calibrated for each channel of the inlet and outlet pumps before the experiments, and they are monitored during the experimental runs to ensure stable flow conditions. The porous medium in the three-dimensional flow-through chamber consists of three layers. It has spatially heterogeneous and anisotropic permeability at the relevant scale for the observation of helical flow [18], but isotropic porosity. Both layer 1 and layer 2 consist of two types of glass beads (Sigmund Lindner, Warmensteinach, Germany), one with a fine grain size of $0.4-0.6 \mathrm{~mm}$ and the other with a coarse grain size of $1.5-2.0 \mathrm{~mm}$. The porosity was determined gravimetrically, and a value of 0.4 was found for both the fine and the coarse material. The architecture of the porous medium was obtained by alternating angled stripes of high and low hydraulic conductivity in layer 1 and layer 2 (Fig. 1), and it was designed to achieve a simplified representation of herringbone crossstratification, a pattern observed in subsurface sedimentary depositional environments [19]. Therefore, the porosity is invariant through the medium, but the permeability, and thus the hydraulic conductivity, varies from one stripe to the next. The hydraulic conductivity within the stripes is locally isotropic; its value was calculated from the grain size following Hazen [20]. A metal frame was used to achieve this arrangement during the filling of each layer and then removed. The height of the two layers was identical $(3.08 \mathrm{~cm})$. A homogeneous layer of fine material $(0.4-0.6 \mathrm{~mm})$ saturated for a thickness of $1.54 \mathrm{~cm}$ was placed on top of layer 2. Such a layer was needed to maintain water-saturated conditions in the coarse glass beads in layer 1 and layer 2 . The average seepage velocity in the porous medium was $3 \mathrm{~m} /$ day for all experimental runs, which leads to a maximum of grain-Péclet number (i.e., $\mathrm{Pe}=v d / D_{\text {aq }}$, where $v$ is the seepage velocity, $d$ is the 


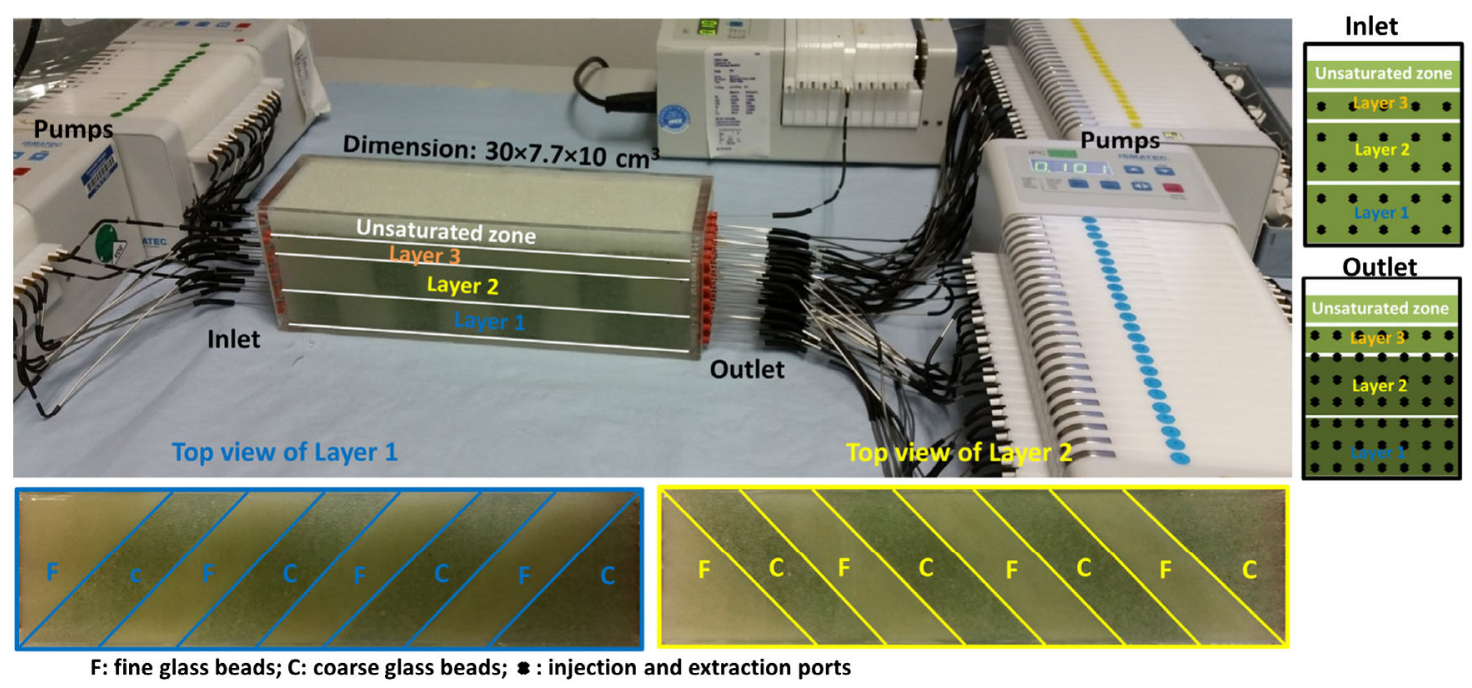

FIG. 1 (color online). Photograph of the experimental setup: Top view of the two layers with alternate stripes of fine and coarse material used to build the heterogeneous anisotropic porous medium, and side views of the inlet and the outlet with marked injection and extraction ports, respectively.

grain diameter, and $D_{\text {aq }}$ is the aqueous diffusion coefficient) of $2.25 \times 10^{3}$, and a maximum grain-Reynolds number (i.e., $\operatorname{Re}=q d / \nu$, where $q$ is the specific discharge and $\nu$ is the kinematic viscosity) of 1.08 (data derived from the numerical simulations). After establishing steady-state flow conditions, a dilute sodium fluorescein solution with a concentration of $15 \mathrm{mg} / 1$ was injected from a selected inlet port. Three experiments were performed exchanging the tracer injection port. After flushing 8 pore volumes, steadystate transport conditions were achieved and samples were collected at the 49 outlet ports. Fluorescein concentrations at all ports were measured using a UV-spectrometer (Perkin Elmer LS-3B), and the flow rate at each port was determined gravimetrically by weighting the samples and measuring the sampling time.

We performed flow and transport simulations using the numerical code described in Ref. [21] to quantitatively interpret the experimental observations. Flow was calculated according to the governing steady-state flow equation in saturated porous media obtained by combining Darcy's law and the continuity equation:

$$
\nabla \cdot(\mathbf{q}(\mathbf{x}))=\nabla \cdot(-\mathbf{K}(\mathbf{x}) \nabla \phi(\mathbf{x}))=0,
$$

where $\mathbf{x}[L]$ denotes the vector of spatial coordinates, $\mathbf{K}$ $\left[L T^{-1}\right]$ is the hydraulic conductivity tensor (here assumed isotropic as the stripes of different materials are spatially resolved), $\phi[L]$ is the hydraulic head, and $\mathbf{q}\left[L T^{-1}\right]$ is the specific discharge vector as defined by Bear [22]. In our experimental setup the permeabilities and their differences are low; thus, the Brinkman correction term in Darcy's law can be neglected [23]. Equation (1) was solved considering fixed-flux boundary conditions for each injection and extraction port, while no-flow conditions were applied at the other boundaries of the flow-through chamber. Steadystate nonreactive transport was described by the advectiondispersion equation:

$$
\mathbf{v} \cdot \nabla c-\nabla \cdot\left(D_{t} \nabla c\right)=0
$$

where $c\left[M L^{-3}\right]$ is the concentration, $\mathbf{v}=\mathbf{q} / \theta\left[L T^{-1}\right]$ is the seepage velocity vector (also denoted as linear average velocity of water), $\theta[-]$ is the porosity, and $D_{t}\left[L^{2} T^{-1}\right]$ is the transverse dispersion coefficient. For continuous injection under steady-state transport conditions the longitudinal dispersion term in the advection-dispersion equation can be neglected [24]. The accurate description of the transverse dispersion coefficient should properly capture the coupled interaction between the aqueous diffusivity of the solute $\left(D_{\text {aq,fluorescein }}=0.48 \times 10^{-9} \mathrm{~m}^{2} / \mathrm{s}\right)$ and mechanical dispersion $[25,26]$. In this study, we used the nonlinear compound-specific parametrization proposed by Chiogna et al. [27] with the parameters for the materials reported by Ye et al. [28]. A constant injection concentration was applied as a boundary condition for the inlet ports. The fixed concentration was $15 \mathrm{mg} / \mathrm{l}$ for the tracer injection port and $0 \mathrm{mg} / 1$ for all surrounding ports through which pure water was pumped into the flow-through system.

Dilution of steady-state solute plumes along the main flow direction $x$ is quantified using the flux-related dilution index $E_{Q}(x)\left[L^{3} T^{-1}\right][29]$ :

$E_{Q}(x)=\exp \left\{-\int_{\Omega}\left[p_{Q}(x, y, z) \ln p_{Q}(x, y, z)\right] q_{x}(x, y, z) d A\right\}$,

where $\Omega$ is the cross-section perpendicular to the main flow direction, $q_{x}(x, y, z)\left[L T^{-1}\right]$ is the specific discharge 
component in the longitudinal direction $x$, and $p_{Q}(x, y, z)$ $\left[T L^{-3}\right]$ is the flux-weighted probability density function of the solute mass, defined as

$$
p_{Q}(x, y, z)=\frac{c(x, y, z)}{\int_{\Omega} c(x, y, z) q_{x}(x, y, z) d A} .
$$

The metric $E_{Q}$ was derived in analogy to the (volumerelated) dilution index $E\left[L^{3}\right]$ [30] and has been used in both Darcy and Stokes flows [31]. It represents the exponential of the Shannon or information entropy, which is a measure of disorder [32] of the solute mass flux and quantifies how a given solute mass flux is distributed over the water flux transporting the solute at a given longitudinal position $x$. While Eq. (3) contains the logarithm of a dimensional property (here $p_{Q}$ ), it is dimensionally correct and may be written as the limit of a discrete exponential of the entropy where all terms in exponents and logarithms are dimensionless [30]. $E_{Q}$ was calculated at the inlet and at the outlet according to the experimental measurements of concentration and flow rate, while it was computed at all cross sections along the travel distance in the numerical simulations.

Figure 2 shows the tracer concentrations at the outlet of the flow-through setup for the three experiments performed injecting the tracer solution from three different inlet ports.
The results are shown as normalized concentrations measured at the outlet ports (i.e., measured values normalized by the concentration of fluorescein in the tracer solution). The two-dimensional maps showing the experimental distribution of fluorescein at the outlet [Figs. 2(a)-2(c)] are obtained by interpolating the concentration measurements at the 49 outlet ports with the cubic interpolation algorithm implemented in Matlab. Figures 2(d)-2(f) show the concentration distribution computed in the numerical simulations of the different experiments. Notice that the results at the outlet show that the plume location, the peak concentration, as well as the shape of the plume changed considerably in the different experiments. Because of the flow field in the three-dimensional setup, the location of the plume at the outlet significantly deviates from the position of the inlet port used to inject the tracer solution and indicated by a white cross in Fig. 2. Also, the peak concentrations observed at the outlet differ depending on the port chosen to inject the tracer. Figure 2(b) shows the highest peak concentrations while Fig. 2(c) shows the lowest value. Furthermore, the shape of the plume at the outlet cross section is also different in the three cases. These effects indicate that the flow field exerts a significant control on tracer transport, on the deformation of the material surface of the plume, and on plume dilution. Figures 2(g)-2(i) show the comparison of the normalized
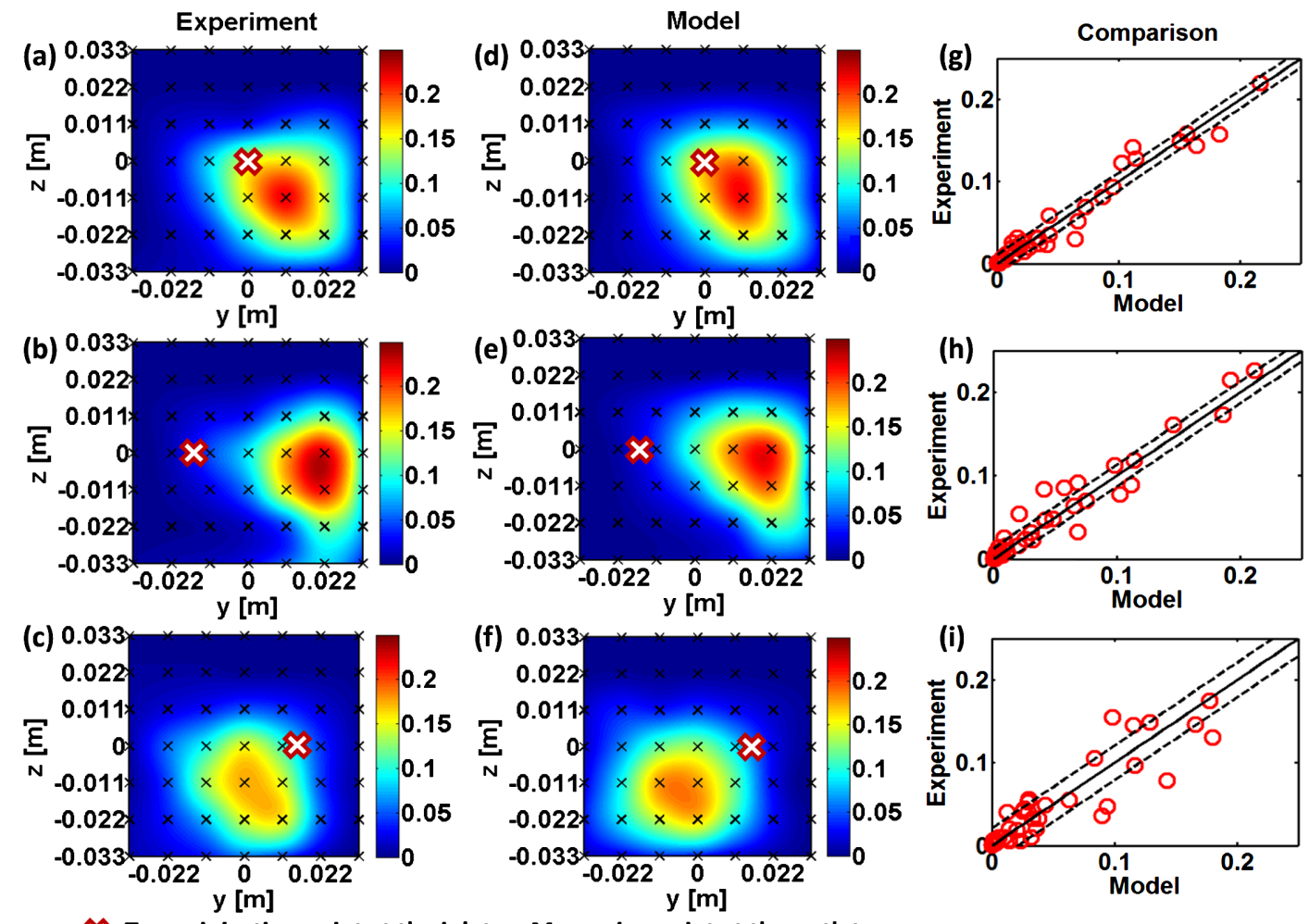

X: Tracer injection point at the inlet; $x$ : Measuring point at the outlet

FIG. 2 (color online). Results of normalized concentration distributions at the outlet of the heterogeneous anisotropic flow-through system for the three experiments performed using different tracer injection ports. (a)-(c) The experimental results. (d)-(f) The model results. (g)-(i) The comparison between observed and simulated normalized concentrations. 
concentrations measured at the outlet ports and the values obtained from the purely forward simulation of the different flow-through experiments. The experimental error $\varepsilon[-]$ is estimated applying the reduced $\chi^{2}$ test:

$$
\chi^{2}=\frac{1}{n_{\text {ports }}} \sum_{n=1}^{n_{\text {ports }}} \frac{\left(c_{\text {meas }}-c_{\text {simu }}\right)^{2}}{\varepsilon^{2}}
$$

such that $\chi^{2}$ meets its expected value of unity. Here, $n_{\text {ports }}$ [-] is the number of the outlet ports (49 in our setup), $c_{\text {meas }}$ [-] is the measured normalized concentration at each port, and $c_{\text {simu }}[-]$ is the simulated normalized concentration at each port. The experimental error estimated according to Eq. (5) is shown as dashed lines in Figs. 2(g)-2(i). The forward model captures the main flow and transport processes occurring in the experimental setup. In particular, a very good agreement was found between the measured and simulated results in the first two experiments, whereas the deviations were larger in the third flow-through experiment.

The simulations are instrumental to visualize and explain the laboratory results and, in particular, the remarkable deviation of the peak concentration location at the outlet with respect to the inlet injection port. To further illustrate this point, numerical simulations of streamlines, determined by particle tracking [33] according to Eq. (1), are shown in Figs. 3(a)-3(c). The streamlines (black lines) provide a clear image of the complex velocity field in the three-dimensional setup. Streamlines starting at the source twist along the travel distance, assuming a helical shape, and terminate with remarkable shifts compared to the inlet location. Such behavior is caused by the interaction of macroscopically anisotropic hydraulic conductivity with changing orientation in the different layers and the no-flow boundary conditions [18]. Figure 3 also shows the occurrence of deformed isosurfaces [color surfaces in Figs. 3(a)-3(c)] with constant hydraulic head $\phi$. These isosurfaces are not perpendicular to the streamlines, indicating therefore the occurrence of nontrivial Lamb surfaces and hence nonzero helicity density.

The helical flow occurring in our experimental setup affects mixing and dilution processes by deforming the material surface of the plume, causing an increase of diffusive and transverse dispersive fluxes, thus increasing the probability of mass exchange between two streamlines. As a result, plume dilution is considerably enhanced. The flux-related dilution index, quantifying plume dilution at different cross sections, is shown in Figs. 3(d)-3(f). The solid red lines show the simulated behavior of the
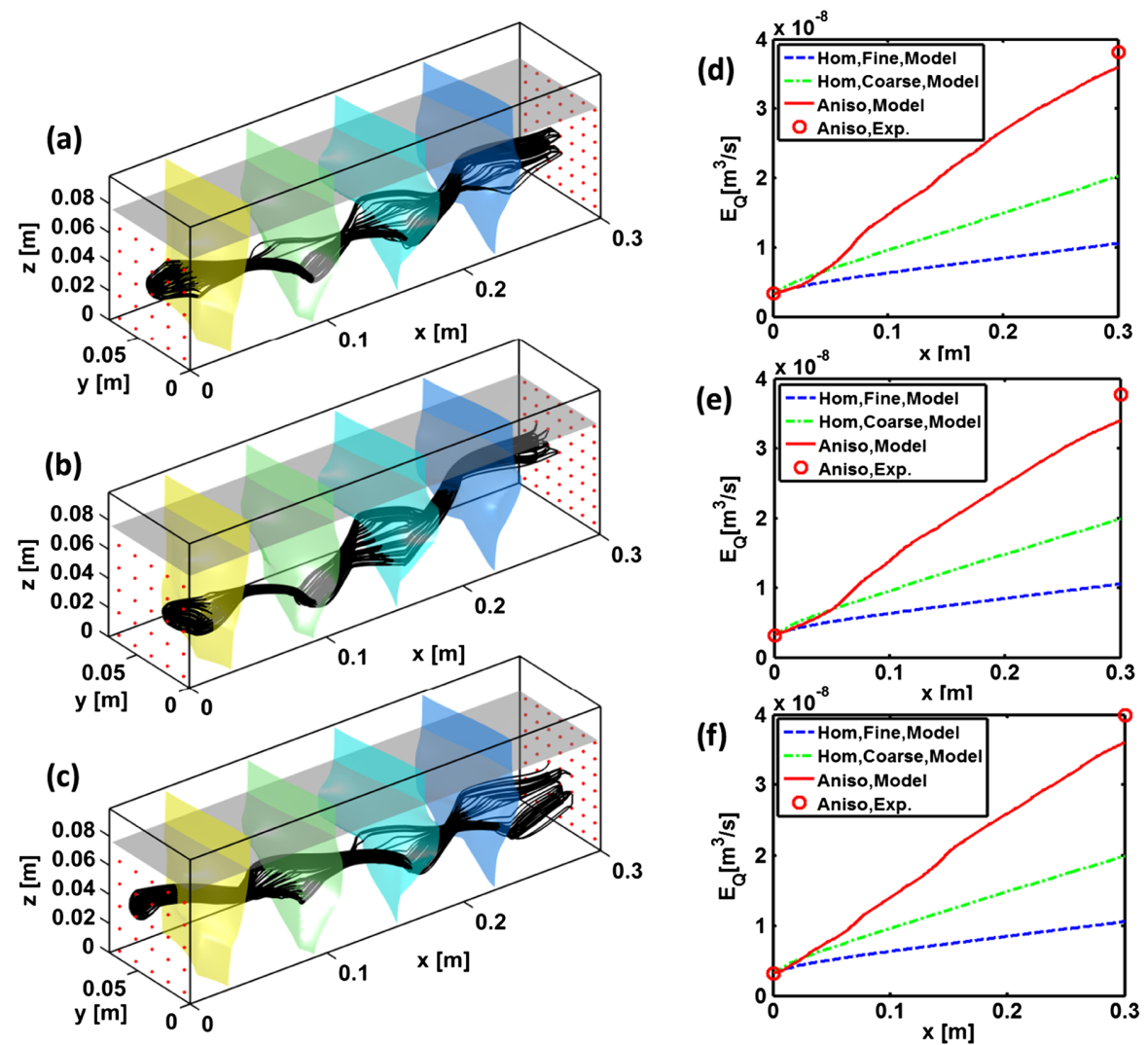

FIG. 3 (color online). (a)-(c) Streamlines traced from the inlet injection ports show a helical behavior; black lines: streamlines; colored surfaces: isosurfaces representing points with constant hydraulic head $\phi$; grey surfaces: water level. (d)-(f) Flux-related dilution index observed (circles) and simulated (lines) at cross-sections along the travel distance in the three flow-through experiments. 
flux-related dilution index along the main flow direction in the flow-through system, whereas the red circles represent the experimental values measured at the inlet and at the outlet of the setup. The monotonic increase of $E_{Q}$ with the travel distance captures the spatial increase of the entropy of the plume due to the lateral diffusive and dispersive processes that distribute the solute mass flux over an increasing water flux. The increase of plume dilution between the inlet and the outlet of the setup is remarkable, and the values of $E_{Q}$ at the outlet of the setup in the three flow-through experiments are in the range $3.5 \times 10^{-8}-4.0 \times 10^{-8} \mathrm{~m}^{3} / \mathrm{s}$ and show more than a tenfold increase compared to the values at the inlet. To assess the contribution of helical flow to plume dilution, it is interesting to compare the outcomes obtained for the heterogeneous anisotropic domains with homogeneous porous media. To this end, the flux-related dilution index has been also computed using the numerical model for two homogeneous porous media, entirely packed with fine glass beads (dashed blue lines in Fig. 3) or with coarse glass beads (dash-dotted green lines in Fig. 3), respectively. Both the values of $E_{Q}$ and its rate of increase show that plume dilution in the heterogeneous and anisotropic porous medium is considerably larger compared to the homogeneous domains. In particular, the values at the outlet show an average dilution enhancement in the anisotropic setups of $235 \%$ and $75 \%$ compared to the homogeneous fine and coarse grain size scenarios, respectively. This indicates the relevance of the occurrence of helical flow in porous media and its remarkable effect on solute transport and mixing processes.

In conclusion, we provide first experimental evidence of helical flows in porous media. Such flows with twisting streamlines have a large impact on the dilution enhancement of steady-state solute plumes. Since this is the rate limiting step for mixing-controlled reactions, helical flow will lead to strongly enhanced chemical reactions in plumes. The outcomes of this study extend the findings of complex flows and associated mixing processes carried out for Stokes flows in microchannels [6,34] to Darcy flows in porous media, occurring at larger scales. These results are relevant for an improved understanding of dilution and mixing processes occurring in engineered systems such as packed bed reactors as well as in natural systems such as geologic formations and aquifer systems.

This work was supported by the DFG (Deutsche Forschungsgemeinschaft, Grants No. RO 4169/3-1 and No. CI-26/11-1). The authors thank four anonymous reviewers for their constructive comments.

* Corresponding author. masro@env.dtu.dk

[1] V. Hessel, H. Löwe, and F. Schönfeld, Chem. Eng. Sci. 60, 2479 (2005).
[2] G. Chiogna, D. L. Hochstetler, A. Bellin, P. K. Kitanidis, and M. Rolle, Geophys. Res. Lett. 39, L20405 (2012).

[3] J. J. Hidalgo, J. Fe, L. Cueto-Felgueroso, and R. Juanes, Phys. Rev. Lett. 109, 264503 (2012).

[4] M. Icardi, G. Boccardo, D. L. Marchisio, T. Tosco, and R. Sethi, Phys. Rev. E 90, 013032 (2014).

[5] J.M. Ottino, The Kinematics of Mixing (Cambridge University, Cambridge, England, 1989).

[6] A. D. Stroock, S. K. W. Dertinger, A. Ajdari, I. Mezić, H. A. Stone, and G. M. Whitesides, Science 295, 647 (2002).

[7] B. Bijeljic, P. Mostaghimi, and M. J. Blunt, Phys. Rev. Lett. 107, 204502 (2011).

[8] J. C. Giddings, Dynamics of Chromatography (Dekker, New York, 1965).

[9] E. Villermaux, A. D. Stroock, and H. A. Stone, Phys. Rev. E 77, 015301 (2008).

[10] H. K. Moffatt, Proc. Natl. Acad. Sci. U.S.A. 111, 3663 (2014).

[11] P. de Anna, T. Le Borgne, M. Dentz, A. M. Tartakovsky, D. Bolster, and P. Davy, Phys. Rev. Lett. 110, 184502 (2013).

[12] P. de Anna, J. Jimenez-Martinez, H. Tabuteau, R. Turuban, T. Le Borgne, M. Derrien, and Y. Méheust, Environ. Sci. Technol. 48, 508 (2014).

[13] F. P. J. de Barros, M. Dentz, J. Koch, and W. Nowak, Geophys. Res. Lett. 39, L08404 (2012).

[14] S. Torquato, Random Heterogeneous Materials (SpringerVerlag, New York, 2002).

[15] G. Sposito, Water Resour. Res. 30, 2395 (1994).

[16] M. Bakker and K. Hemker, Adv. Water Resour. 27, 1075 (2004).

[17] G. Chiogna, M. Rolle, A. Bellin, and O.A. Cirpka, Adv. Water Resour. 73, 134 (2014).

[18] G. Chiogna, O. A. Cirpka, M. Rolle, and A. Bellin, Water Resour. Res. 51, 261 (2015).

[19] G. Nichols, Sedimentology and Stratigraphy (Wiley-Blackwell, Chichester, 2009).

[20] A. Hazen, Annu. Rep. State Board of Health Mass 24, 541 (1892).

[21] O. A. Cirpka, G. Chiogna, M. Rolle, and A. Bellin, Water Resour. Res. 51, 241 (2015).

[22] J. Bear, Dynamics of Fluids in Porous Media (Dover, New York, 1972).

[23] H. C. Brinkman, Appl. Sci. Res. Sect. A 1, 27 (1949).

[24] O. A. Cirpka, F. P. J. de Barros, G. Chiogna, M. Rolle, and W. Nowak, Water Resour. Res. 47, W06515 (2011).

[25] U. M. Scheven, Phys. Rev. Lett. 110, 214504 (2013).

[26] U. M. Scheven, S. Khirevich, A. Daneyko, and U. Tallarek, Phys. Rev. E 89, 053023 (2014).

[27] G. Chiogna, C. Eberhardt, P. Grathwohl, O. A. Cirpka, and M. Rolle, Environ. Sci. Technol. 44, 688 (2010).

[28] Y. Ye, G. Chiogna, O. A. Cirpka, P. Grathwohl, and M. Rolle, J. Contam. Hydrol. 172, 33 (2015).

[29] M. Rolle, C. Eberhardt, G. Chiogna, O. A. Cirpka, and P. Grathwohl, J. Contam. Hydrol. 110, 130 (2009).

[30] P. K. Kitanidis, Water Resour. Res. 30, 2011 (1994).

[31] M. Rolle, G. Chiogna, D. L. Hochstetler, and P. K. Kitanidis, J. Contam. Hydrol. 153, 51 (2013).

[32] E. T. Jaynes, Phys. Rev. 106, 620 (1957).

[33] D. W. Pollock, Ground Water 26, 743 (1988).

[34] C. Simonnet and A. Groisman, Phys. Rev. Lett. 94, 134501 (2005). 